\title{
Inverse Compton scattering X-ray source for research, industry and medical applications
}

Benjamin Hornberger, Jack Kasahara, Ronald Ruth, Rod Loewen, John Khaydarov

Benjamin Hornberger, Jack Kasahara, Ronald Ruth, Rod Loewen, John Khaydarov, "Inverse Compton scattering X-ray source for research, industry and medical applications," Proc. SPIE 11886, International Conference on XRay Lasers 2020, 1188609 (8 July 2021); doi: 10.1117/12.2591977

SPIE. Event: XVII International Conference on X-Ray Lasers, 2020, Online Only 


\title{
Inverse Compton Scattering X-ray Source for Research, Industry and Medical Applications
}

\author{
Benjamin Hornberger*, Jack Kasahara, Ronald Ruth, Rod Loewen, John Khaydarov \\ Lyncean Technologies, Inc., 47633 Westinghouse Dr, Fremont, CA 94539, USA
}

\begin{abstract}
There is a large performance gap between conventional, electron-impact X-ray sources and synchrotron radiation sources. An Inverse Compton Scattering (ICS) source can bridge this gap by providing a narrow-band, high-flux and tunable Xray source that fits into a laboratory. It works by colliding a high-power laser beam with a relativistic electron beam, in which case the energy of the backscattered photons is in the X-ray (or gamma-ray) regime. Here we present a new conceptual design for an ICS source that is more than two orders of magnitude brighter than the Lyncean Compact Light Source (CLS) currently in user operation. Depending on configuration, this next generation CLS covers an X-ray energy range of about 30-90 keV, or $60-180 \mathrm{keV}$. It will provide X-ray flux of up to $4 \times 10^{12}$ photons/s within a beam divergence of $4 \mathrm{mrad}$ and a bandwidth of around $10 \%$. This is well-suited for micro-CT imaging of millimeter-sized samples at micron resolution, with a flux density similar to some high-energy synchrotron beamlines. The beam properties of the new design are also compatible with narrower bandwidth, focused beam applications such as high-energy diffraction. We discuss the novel concepts applied to the design of this X-ray source as well as the resulting beam properties. We present application examples in the areas of imaging, diffraction, and radiotherapy where this system can approach or match the performance of synchrotron beamlines. This will allow transferring many research, industrial and medical applications from the synchrotron, where capacity and access are limited, to a local lab or clinic.
\end{abstract}

Keywords: X-ray source, inverse Compton scattering, compact light source, table-top synchrotron

\section{INTRODUCTION}

There is a large performance gap between conventional, electron-impact X-ray sources and synchrotron radiation sources. Electron-impact sources are compact with low to moderate cost, making them accessible for a wide range of X-ray applications and laboratories. On the other hand, their flux and brightness are limited, limiting their measurement speed and/or data quality on many types of samples. Furthermore, their spectrum, consisting of fixed-energy emission lines (depending on the choice of target material) on top of a continuum of bremsstrahlung (with a cutoff energy given by the acceleration voltage), makes them less-well suited for applications requiring energy tunability and a high degree of monochromaticity. Such applications are well-served by synchrotron radiation facilities, which provide high-flux, highbrightness beams that are tunable and can be monochromatized to a high degree. However, synchrotrons are large and expensive to build and operate, which is why they are typically operated as government-funded facilities and can provide only a limited amount of beamtime.

An Inverse Compton Scattering (ICS) X-ray source ${ }^{1,2}$ can bridge this gap by providing a narrow-band, high-flux and tunable X-ray source that fits into a laboratory at a cost of a few percent of a large synchrotron facility. It works by colliding a high-power laser beam with a relativistic electron beam, in which case the energy of the backscattered photons is in the $\mathrm{X}$-ray (or gamma-ray) regime. So far, the only ICS source in regular user operation is the Munich Compact Light Source (MuCLS), ${ }^{3,4}$ a combination of Lyncean Technologies' commercially available Compact Light Source (CLS) ${ }^{5}$ and a beamline with two endstations built by researchers at the Technical University of Munich. ${ }^{6}$ The application focus of the MuCLS is biomedical imaging of centimeter-sized samples in the $15-35 \mathrm{keV}$ energy range, well-matched to the beam properties of the first generation Lyncean CLS with $\sim 4$ mrad divergence and spectral bandwidth of 3-5\%. For a summary of application examples from the MuCLS incl. further references, please see Ref. ${ }^{6}$.

Following design principles developed for a narrow-band gamma-ray source for the ELI-NP project (www.eli-np.ro), we have recently developed a concept for a next generation CLS that will be more than two orders of magnitude brighter than the source in operation at the MuCLS. With two different configurations (laser wavelengths), it can cover an X-ray energy

*benjamin_hornberger@lynceantech.com; phone 16503208300 x427; www.lynceantech.com

International Conference on X-Ray Lasers 2020, edited by Davide Bleiner,

Proc. of SPIE Vol. 11886, $1188609 \cdot$ - () 2021 SPIE - CCC code:

0277-786X/21/\$21 - doi: 10.1117/12.2591977

Proc. of SPIE Vol. 11886 1188609-1 
range of about 30-90 keV, or 60-180 keV (the lower end of the energy range might be reduced further depending on optimization of the design). This shift towards higher energies changes the application focus of the source towards studies of larger samples and denser materials, enabling use cases in geo- and materials science as well as biomedical imaging of larger samples. Furthermore, the energy range and higher flux of this source provides a pathway for transferring radiotherapy applications currently performed at synchrotrons into a clinical setting.

The performance (flux and brightness) of synchrotron beamlines typically drops towards higher energies. By contrast, ICS sources perform better at higher energies because the opening angle of the photon beam scales inversely with the electron beam energy: $\theta_{0}=1 / \gamma$, where $\theta_{0}$ is the opening angle, containing $1 / 2$ of the total photon flux, and $\gamma$ is the electron beam energy in units of electron rest mass. Consequently, the next generation CLS is expected to reach the performance level of some synchrotron beamlines at higher energies. This is qualitatively illustrated in Fig. 1, and a more quantitative comparison to select beamlines is provided in Sec. 3 .

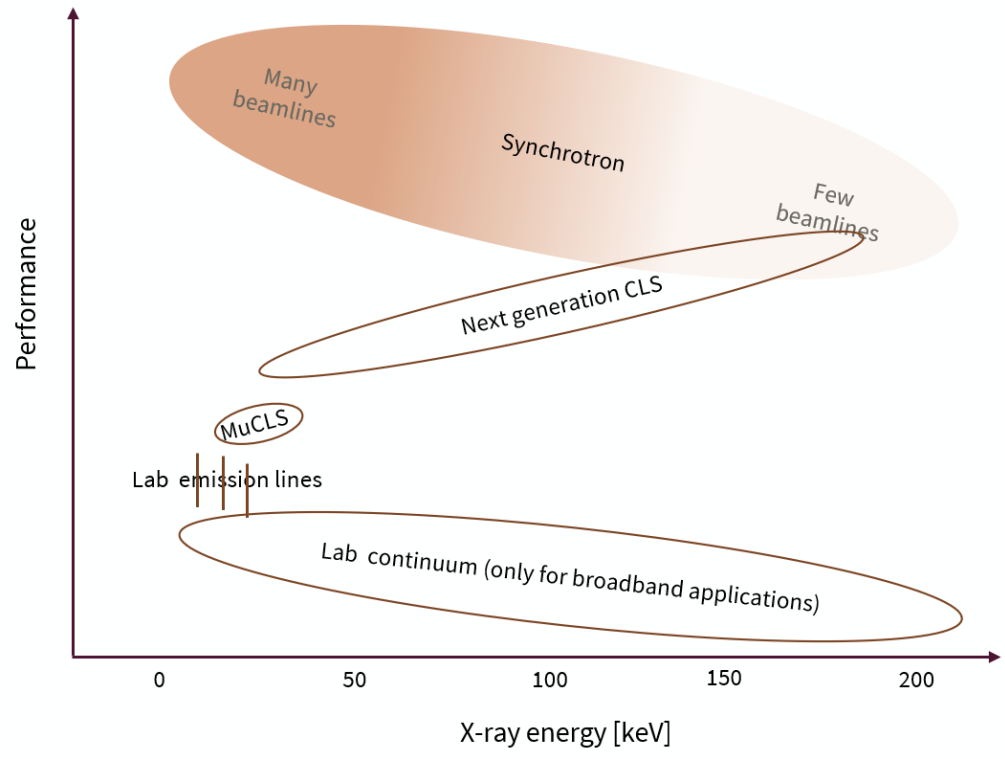

Figure 1. Qualitative comparison of different types of X-ray sources, plotting a generic performance parameter (e.g., brightness, or flux for a particular application) as a function of X-ray energy. While the MuCLS operates at lower energies $(15-35 \mathrm{keV})$, the next generation CLS will operate at substantially higher energies (up to $180 \mathrm{keV}$ ) and is expected to match the performance of some synchrotron beamlines at higher energies.

In the following sections, we first briefly review the principles of inverse Compton scattering. We then present the design and target performance parameters of the next generation CLS, followed by a quantitative comparison to some laboratory sources and synchrotron beamlines. Finally, we discuss some applications well-suited for this X-ray source.

\section{INVERSE COMPTON SCATTERING}

We have previously described the principles of inverse Compton scattering, ${ }^{5}$ which we briefly summarize here. In the wave picture, inverse Compton scattering can be explained as analogous to undulator radiation in a synchrotron facility, where the electromagnetic field of a laser beam generates X-rays in the same way as the magnetic field of a permanent magnet undulator. In the particle picture, it can be explained by backscattering of a laser photon off a relativistic electron as illustrated in Fig. 2a. The energy of the backscattered photon is upshifted into the X-ray regime by energy transfer from the electron. The peak energy (for head-on collision with $\theta_{i}=180^{\circ}$ and $\theta_{f}=0^{\circ}$, referring to the angles denoted in Fig. $2 \mathrm{a})$, the energy $E_{x}$ of the backscattered photon is given by

$$
E_{x}=4 \gamma^{2} E_{L}
$$

where $\gamma$ is the Lorentz factor (electron energy in units of electron rest mass) and $E_{L}$ is the energy of the incident laser photon. The energy of photons scattered at larger angles $\left(\left|\theta_{f}\right|>0\right)$ is lower as explained in Ref. ${ }^{5}$, contributing to the finite 
bandwidth of an inverse Compton scattering X-ray source. Fig. $2 \mathrm{~b}$ shows the peak X-ray energy for the next generation CLS design presented in Sec. 3.

(a)

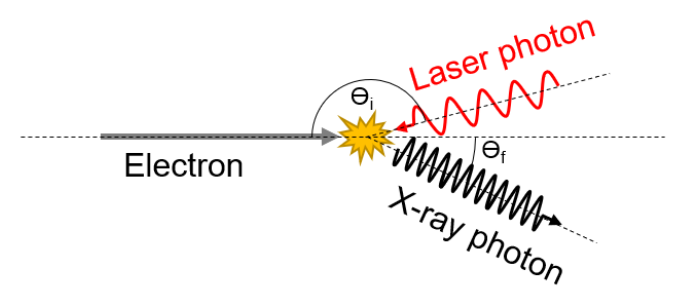

(b)

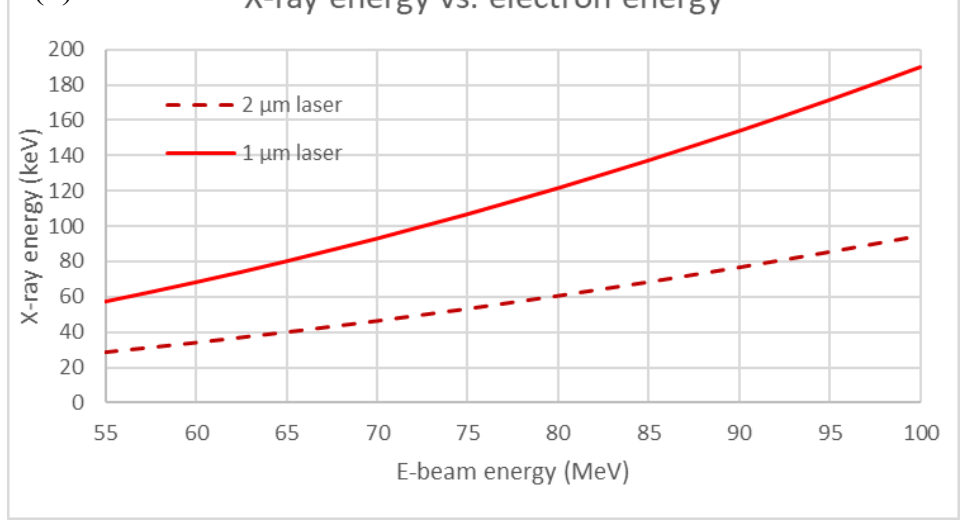

Figure 2. (a) Principle of inverse Compton scattering. A laser photon scatters off a relativistic electron. The backscattered photon's energy is upshifted into the X-ray regime. (b) Peak energy of backscattered photons as function of electron beam energy for laser wavelengths of $1 \mu \mathrm{m}$ and $2 \mu \mathrm{m}$ and the electron energy range considered for the next generation CLS.

\section{NOVEL DESIGN FOR AN INVERSE COMPTON SCATTERING SOURCE}

Our work on developing a state-of-the-art ICS-based gamma-ray source with narrow bandwidth and high spectral density for the ELI-NP project (www.eli-np.ro) has led to new approaches for optimizing an ICS source's brightness: narrow bandwidth, high spectral density, and narrow beam divergence. Here we present a conceptual design for a next generation CLS that is more than two orders of magnitude brighter than the existing CLS design in operation at the MuCLS. The principle is primarily based upon an increase in electron beam energy from $45 \mathrm{MeV}$ to a maximum of $100 \mathrm{MeV}$, which yields a lower emittance electron beam, decreased damping time, and higher storage ring current through charge accumulation (top-up mode). The lower emittance, combined with more parallel electron and laser beams, leads to a lower divergence and narrower bandwidth X-ray beam.

The ICS X-rays have higher energy due to the higher e-beam energy but can be shifted back down in energy by utilizing an optical cavity with a longer wavelength laser $(2 \mu \mathrm{m}$ instead of $1 \mu \mathrm{m}$ in the existing CLS). Optical enhancement cavities in the near-infrared region $(\sim 1.5 \mu \mathrm{m})$ and short-wave infrared $(3-5 \mu \mathrm{m})$ spectral ranges have been extensively used in spectroscopic applications like Cavity Ring-Down Spectroscopy (CRDS). ${ }^{7,8}$ These examples demonstrate that technologies of low-loss optical coatings and materials exist and can be used for the $2 \mu \mathrm{m}$ cavity. Moreover, short-pulse sources with the wavelengths near $2 \mu \mathrm{m}$ have seen considerable progress in research and productization during the last decade. ${ }^{9,10}$

\subsection{Beam parameters}

The target beam parameters of the next generation CLS are presented in Table 1 in comparison to the source in operation at the MuCLS. The increase in electron-beam energy to $100 \mathrm{MeV}$ shifts the X-ray photon energy up significantly, even when utilizing a longer-wavelength $(2 \mu \mathrm{m})$ laser. This design is expected to achieve a brightness of more than 2.5 orders of magnitude higher than the MuCLS at the respective peak energy. While we currently expect to achieve an X-ray energy as low as $30 \mathrm{keV}$ with a $2 \mu \mathrm{m}$ laser, this limit might be reduced somewhat through further optimization of the design.

With this increase in brightness, it is now practical to utilize the tradeoff of beam divergence, flux and bandwidth that is possible with ICS sources. This is illustrated in Fig. 3 and quantified in Table 1. ICS generates a cone of X-rays, the usable portion of which is defined by an aperture. If the highest flux or large beam size are desired, a large aperture is used (typical values are several milliradians). This is useful, for example, for direct-beam imaging of large samples. However, because ICS photons scattered at larger angles have a lower energy, a large beam divergence increases the bandwidth of the X-ray beam. If narrow bandwidth is desired, a smaller beam divergence can be used. Smaller beam size is also preferred for focused beam applications, since the achievable aperture with X-ray optics is limited in particular at higher energies. 
Table 1. Target beam parameters of the next generation CLS. ${ }^{(*)}$ The lower end of the energy range might be reduced somewhat through further optimization of the design.

\begin{tabular}{|c|c|c|c|c|c|}
\hline & MuCLS & \multicolumn{4}{|c|}{ Next generation CLS target performance } \\
\hline Stored electron energy $(\max )[\mathrm{MeV}]$ & $\sim 45$ & \multicolumn{4}{|l|}{$\sim 100$} \\
\hline Optical cavity wavelength $[\mu \mathrm{m}]$ & 1 & \multicolumn{2}{|l|}{2} & \multicolumn{2}{|l|}{1} \\
\hline $\mathrm{X}$-ray energy range $[\mathrm{keV}]$ & $15-35$ & \multicolumn{2}{|l|}{$\sim 30^{(*)}-90$} & \multicolumn{2}{|c|}{$\sim 60^{(*)}-180$} \\
\hline Brightness [ph/(s mrad $\left.\left.\mathrm{mm}^{2} 0.1 \% \mathrm{BW}\right)\right]$ & $\sim 1 \times 10^{10} @ 35 \mathrm{keV}$ & \multicolumn{2}{|c|}{$\sim 4 \times 10^{12} @ 90 \mathrm{keV}$} & \multicolumn{2}{|c|}{$\sim 4 \times 10^{12} @ 180 \mathrm{keV}$} \\
\hline Divergence [mrad] & 4 & 1 & 4 & 1 & 4 \\
\hline Bandwidth (FWHM) & $3-5 \%$ & $1.5-2.5 \%$ & $6-15 \%$ & $1.5-2.5 \%$ & $6-15 \%$ \\
\hline Flux@ max energy [ph/s] & $\sim 3 \times 10^{10}$ & $\sim 4 \times 10^{11}$ & $\sim 4 \times 10^{12}$ & $\sim 4 \times 10^{11}$ & $\sim 4 \times 10^{12}$ \\
\hline
\end{tabular}

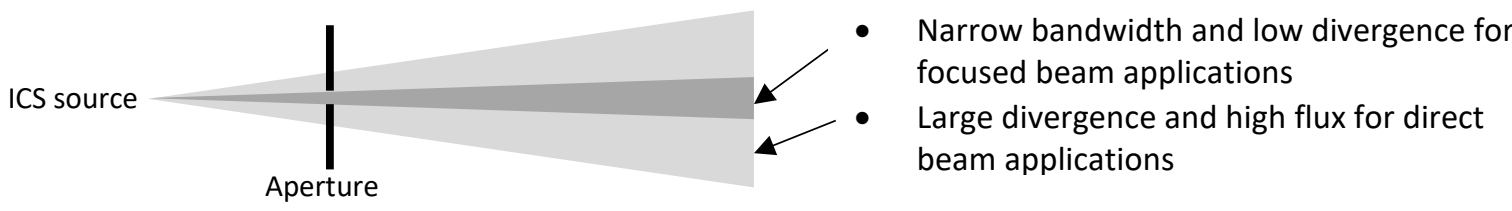

Figure 3. Illustration of the divergence, flux, and bandwidth tradeoff for inverse Compton scattering sources. The ICS source generates a cone of X-rays. An aperture defines the part of that cone that is utilized. Utilizing a large divergence provides the highest flux and largest beam size, but also a larger bandwidth. With a smaller aperture, the bandwidth can be reduced at the expense of flux. Smaller beams are also generally easier to focus using X-ray optics.

\subsection{Conceptual design}

A conceptual design of the instrument is shown in Fig. 4. Electron bunches are generated in the photocathode illuminator and accelerated to their target energy in the electron beam injector (linear accelerator). The electron bunches are then injected into the storage ring where they circulate at a rate of approximately $25 \mathrm{MHz}$. Charge losses are compensated by frequent top-up injection. One leg of the storage ring is shared with a low-loss, power-enhancement optical cavity which is fed by a mode-locked laser that is frequency-matched to the storage ring cycle frequency. With a gain of approximately 10,000 , several hundred kilowatts of laser power can be stored in the optical cavity. Laser pulses and electron bunches collide at the interaction point and generate X-rays via inverse Compton scattering.

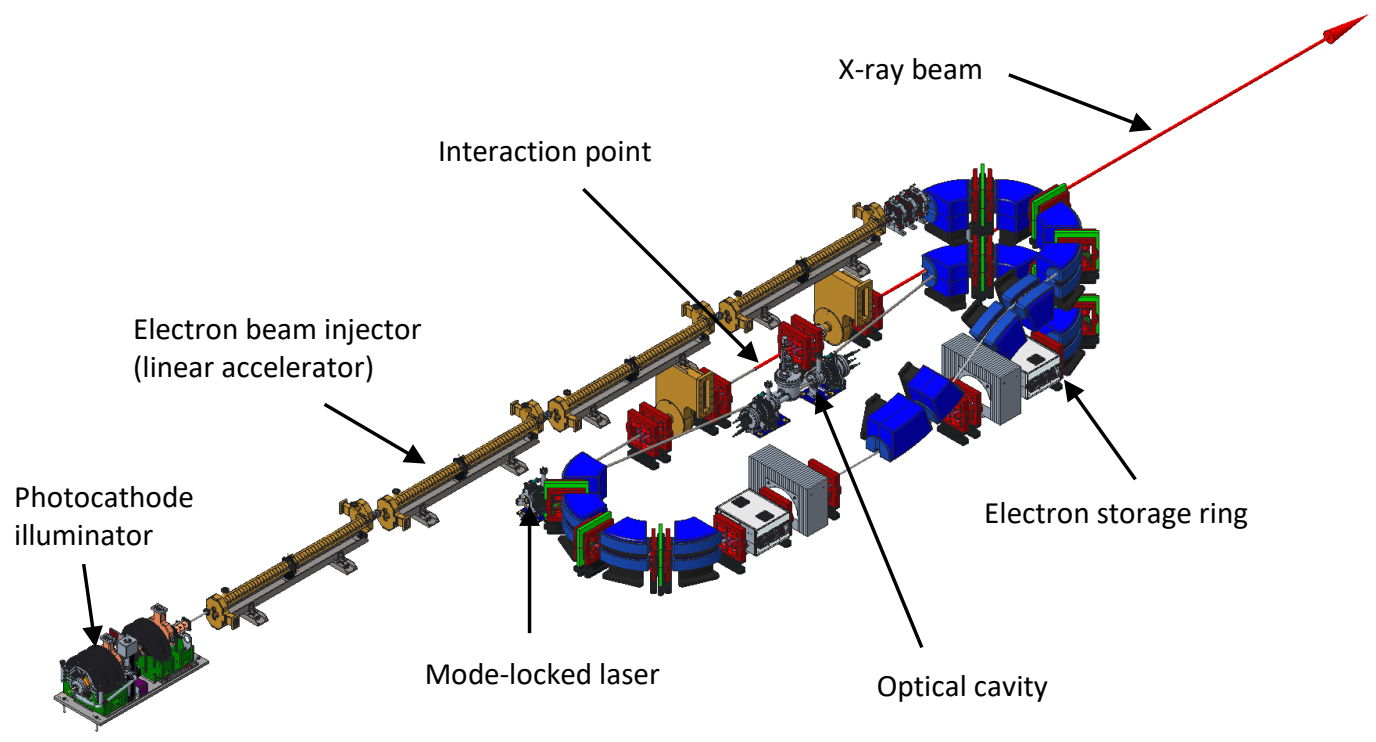

Figure 4. Conceptual CAD design of the next generation CLS. The overall length of the system is approximately $10 \mathrm{~m}$. 


\subsection{Comparison with synchrotron and laboratory sources: Flux density}

Flux density of the X-ray beam is relevant for techniques such as direct-beam imaging or radiotherapy. Here we compare the flux density achievable with the next generation CLS to select synchrotron beamlines and laboratory sources.

For micro-CT imaging, synchrotron beamlines and the CLS operate in a quasi-parallel-beam geometry, where distance from the source is generally fixed and spatial resolution is determined by the detector. This is limited to slightly below 1 $\mu \mathrm{m}$ for state-of-the-art X-ray cameras based on scintillators with optical magnification. Most laboratory-based micro-CT systems operate in a divergent beam geometry with small source spot size, large geometric magnification, and large detector pixels. There are also some systems operating closer to a parallel beam geometry with moderate to large source spot size, low geometric magnification, and small detector pixels. For a divergent beam, flux density scales inversely with the square of the distance from the source, and distance of sample and/or detector from the source can be varied within some constraints in most instruments. Generally, the experimental geometry including source-to-sample and sample-todetector distances is optimized to provide the highest throughput while accommodating the size of the sample and achieving the desired imaging resolution, which is a function of source size, detector resolution and geometry. To provide a realistic flux density comparison, for laboratory sources we have therefore estimated the geometry and corresponding source-to-sample distance to provide imaging resolution around $1 \mu \mathrm{m}$ with a realistic and optimized detector setup. Fig. 5 shows the flux density for the next generation CLS, select synchrotron beamlines and estimates of a liquid metal jet source and a transmission microfocus source.

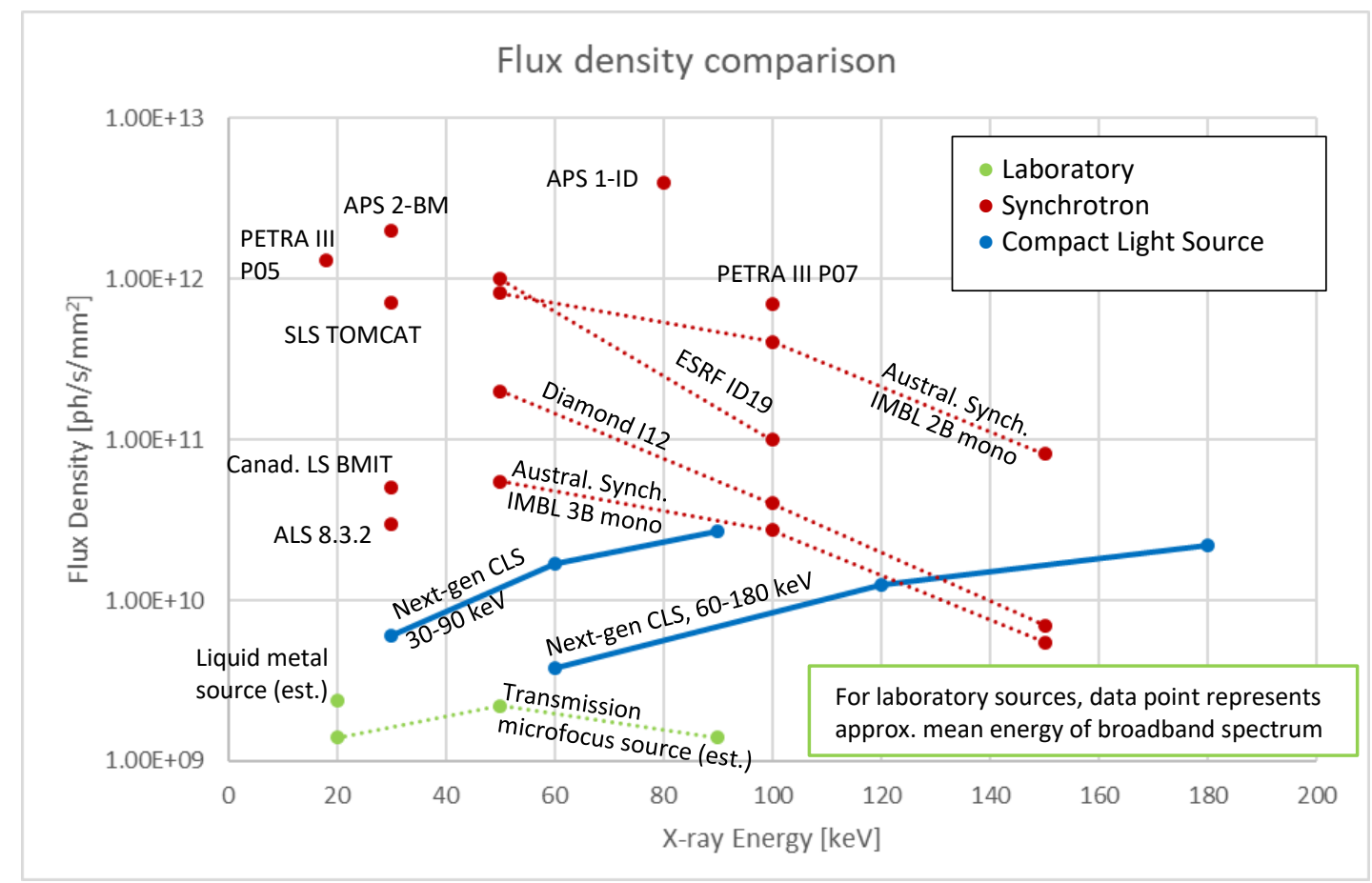

Figure 5. Next generation CLS flux density compared to select synchrotron beamlines and laboratory systems. Synchrotron beamlines are typically tunable; dotted lines represent beamlines for which flux is available at different energies, while individual data points represent beamlines for which flux is only documented at one energy. Flux density for the MuCLS at an imaging distance of $4 \mathrm{~m}$ is on the order of $10^{8} \mathrm{ph} / \mathrm{s} / \mathrm{mm}^{2}$ (based on Table 1 in Ref. ${ }^{6}$ ) and not shown in this plot. See text and Table 2 for details.

With laboratory sources, for direct beam imaging the entire broadband spectrum of the source is used (sometimes filtered with a transmission filter). The data point in the plot represents the approximate mean energy of the spectrum, while the flux density shown includes all the photons in the entire spectrum. Many synchrotron beamlines can operate with different types of monochromators (multilayer monochromators typically providing a bandwidth of order $10^{-2}$, or crystal monochromators providing bandwidth of order $10^{-4}$ ), or even "pink" beams (raw source spectrum filtered by upstream beamline optics). Because for most direct beam applications, bandwidth is not critical, we have used pink beam or multilayer monochromator data where available. The figure quantitatively shows the performance drop of synchrotron 
beamlines, and performance increase of the CLS, towards higher energies, so that the performance of the next generation CLS is comparable to some synchrotron beamlines at those energies. A detailed list of the instruments used for comparison, incl. references, is given in Table 2 in the Appendix.

\subsection{Comparison with synchrotron and laboratory sources: Focused flux}

Here we compare the focused flux achievable with the next generation CLS with select synchrotron beamlines and laboratory systems. Based on the CLS beam properties with source size on the order $100 \mu \mathrm{m}$ FWHM, divergence of a few milliradians and a minimum source-to-optic distance of about $2 \mathrm{~m}$, focusing optics with several millimeters acceptance are needed to make best use of the available flux. Therefore, we consider a multilayer-coated KB-mirror pair in 1:1 focusing geometry that was custom-designed for and has been demonstrated with the first generation CLS from $12-20 \mathrm{keV}$ with $\sim 4 \mathrm{mrad}$ beam acceptance (unpublished). We have analyzed the design for use with the next generation CLS and concluded that it will work with a beam acceptance of $\sim 1 \mathrm{mrad}$ up to $90 \mathrm{keV}$ X-ray energy. X-ray optics such as compound refractive lenses or zone plates have significantly smaller apertures would therefore utilize only a small fraction of the available flux. Therefore we consider focal spot sizes on the order of 100 to a few hundred microns, relevant for applications such as diffraction or moderate spot size scanning probes. This is also comparable to the spot size achievable with standard laboratory sources combined with polycapillary or Montel optics. Focal spot sizes in the micron or submicron range are generally not achievable with meaningful flux with either ICS or standard laboratory sources so are excluded from the comparison here.

Focusing beamlines and laboratory source / focusing optics combinations with spot sizes from sub-100 micron to several hundred microns were researched online. It should be noted that for laboratory sources, the highest energy practically available for focusing is $24 \mathrm{keV}$, the $\mathrm{K}_{\alpha}$ emission line of Indium used in a liquid metal jet source. The Compact Light Source therefore can provide focused beam capabilities at higher energies up to at least $90 \mathrm{keV}$ in a laboratory setting, which would otherwise only be available at synchrotron beamlines.

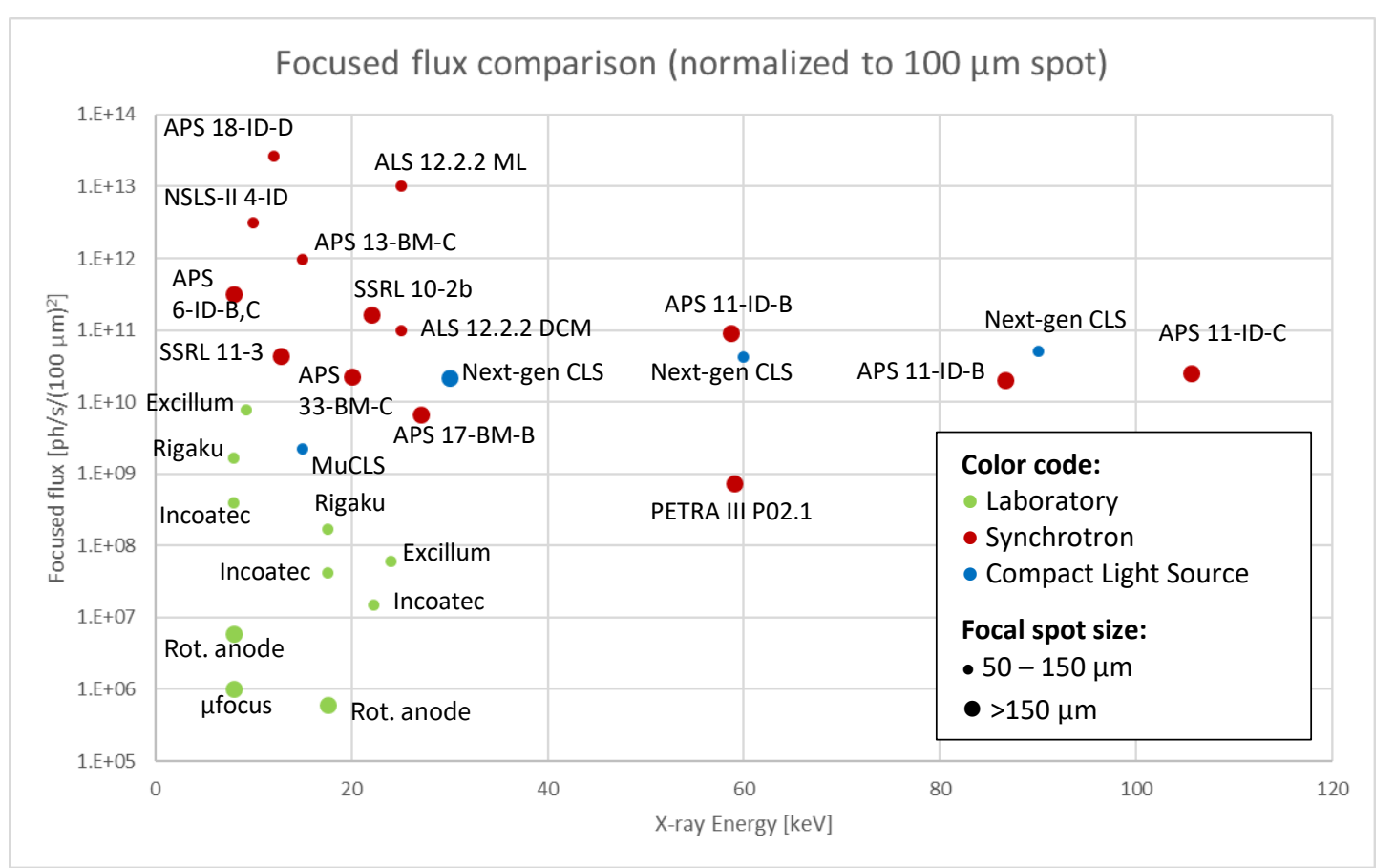

Figure 6. Focused flux of the CLS compared to select synchrotron beamlines and laboratory systems. Synchrotron beamlines are typically tunable, the data points represent the energy documented online. Laboratory sources with focusing optics operate at fixed energy (characteristic emission lines). MuCLS focused flux is estimated for a multilayer-coated KB mirror optics set demonstrated at Lyncean; this optic is not actually available at the MuCLS. See text and Table 3 for details.

Fig. 6 plots focused flux, normalized to $100 \times 100 \mu \mathrm{m}^{2}$, as a function of energy for the next generation CLS, MuCLS, and select synchrotron beamlines and laboratory systems. Focal spot sizes have been categorized into the $50-150 \mu \mathrm{m}$ range 
and $>150 \mu \mathrm{m}$ (round equivalent) and are represented by different-size data points in the plot. As for flux density discussed above, the performance of the next generation CLS is comparable to some synchrotron beamlines in particular at high energies. Details and references are provided in Table 3.

\section{APPLICATIONS}

The next generation Compact Light Source design described in this paper can provide capabilities in particular at higher $\mathrm{X}$-ray energies that are found at only a small number of synchrotron beamlines. The high flux density at higher X-ray energies is ideal for micro-CT imaging of denser samples, for example found in materials or geosciences. It also enables biomedical imaging of larger (several centimeter scale) samples. The high flux density should enable dynamic imaging on time scales not accessible with standard laboratory instruments, on the order of milliseconds for 2D radiography, or seconds for 3D tomography. Fast imaging is also instrumental for high-throughput sample screening, necessary for some industrial applications.

In imaging, energy tunability allows optimizing the contrast for specific sample sizes or compositions. It also enables spectroscopic (K-edge subtraction) imaging, for example using Gadolinium contrast agents (K-edge at $50 \mathrm{keV}$ ). Also, monochromatic beams provide superior image contrast and avoid beam-hardening artifacts typically present with standard laboratory sources, and are more dose-efficient for radiation-sensitive samples.

Utilizing lower beam divergence, the use of X-ray optics becomes feasible even at higher energies, which enables focused beam applications otherwise not possible in a laboratory setting. Examples include diffuse scattering or pair distribution function, which are best performed at energies of 50-100 keV. The substantial increase in data acquisition speed compared to standard laboratory sources enables dynamic diffraction studies otherwise only available at a synchrotron.

Lastly, the high flux density and energy up to $180 \mathrm{keV}$ enables radiotherapy applications with monochromatic beams that are currently only possible at synchrotron radiation facilities. Examples include microbeam radiation therapy (MRT) or metal nanoparticle-based radiotherapy. A compact source with appropriate beam parameters provides a pathway to translating such applications into a clinical setting.

\section{CONCLUSIONS}

The gap between conventional laboratory and synchrotron X-ray sources (see also Sect. 2 -4 [15]) can be bridged by an inverse Compton scattering source, which can provide synchrotron-like beam properties such as high flux, monochromaticity, tunability and partial coherence in a laboratory setting. While synchrotron performance typically drops towards higher energies, ICS sources improve, so that at high energies ICS performance can be comparable to some synchrotron beamlines. An ICS source can thus form the cornerstone of a local, multidisciplinary user facility, serving a wide range of applications and X-ray techniques. This enables spreading synchrotron-like capabilities geographically and building local communities in X-ray instrumentation and applications. Local control of experimental time and access allows overcoming some of the limitations of the standard synchrotron access model, characterized by competitive beamtime proposals and assignment of 2-3-day slots of beamtime with weeks or months of waiting time. For example, it enables iterative and longitudinal studies that require short amounts of measurement time at regular intervals over periods of weeks or months, which is typically not possible at synchrotron beamlines. Difficult-totransport samples (e.g., in vivo, precious, or hazardous samples) can be accommodated more easily at a local facility. Sample pre-screening at a local facility could greatly increase the efficiency of expensive synchrotron or free electron laser facilities. Furthermore, ICS sources provide a pathway to translating some synchrotron applications to clinical or possibly industrial settings. 


\section{APPENDIX}

Tables 2 and 3 below list the synchrotron beamlines and laboratory sources used for the performance comparisons in Secs. 3.3 and 3.4 .

Table 2. Select synchrotron beamlines and laboratory source estimates used for flux density comparison in Sec. 3.3.

\begin{tabular}{|c|c|c|c|c|}
\hline $\begin{array}{l}\text { Beamline / } \\
\text { X-ray } \\
\text { source }\end{array}$ & $\begin{array}{l}\text { Energy } \\
{[\mathrm{keV}]}\end{array}$ & $\begin{array}{l}\text { Flux } \\
\text { density } \\
{\left[\mathrm{ph} / \mathrm{s} / \mathbf{m m}^{2}\right]}\end{array}$ & \multicolumn{2}{|c|}{ Reference / notes } \\
\hline APS 1-ID & 80 & $4.00 \mathrm{E}+12$ & \multicolumn{2}{|c|}{ https://www.aps.anl.gov/Beamlines/Directory/Details?beamline_id=2 } \\
\hline APS 2-BM & 30 & $2.00 \mathrm{E}+12$ & \multicolumn{2}{|c|}{$\begin{array}{l}\text { https://www.aps.anl.gov/Beamlines/Directory/Details?beamline_id=3 } \\
\text { Assume flux is at } 30 \mathrm{keV}\end{array}$} \\
\hline $\begin{array}{l}\text { PETRA III } \\
\text { P05 }\end{array}$ & 18 & $1.30 \mathrm{E}+12$ & \multicolumn{2}{|c|}{$\begin{array}{l}\text { https://photon-science.desy.de/facilities/petra_iii/beamlines/p05_imaging_beamline/ } \\
\text { unified_data_sheet_p05/index_eng.html }\end{array}$} \\
\hline $\begin{array}{l}\text { PETRA III } \\
\text { P07 }\end{array}$ & 100 & $7.00 \mathrm{E}+11$ & \multicolumn{2}{|c|}{$\begin{array}{l}\text { https://photon-science.desy.de/facilities/petra_iii/beamlines/ } \\
\text { p07_high_energy_materials_science/unified_data_sheet_p07/index_eng.html }\end{array}$} \\
\hline ALS 8.3.2 & 30 & $3.00 \mathrm{E}+10$ & \multicolumn{2}{|c|}{$\begin{array}{l}\text { https://als.lbl.gov/beamlines/8-3-2/ } \\
\text { Flux scaled down } 3 x \text { from max at } 10 \mathrm{keV} \text {; multilayer monochromator }\end{array}$} \\
\hline $\begin{array}{l}\text { SLS } \\
\text { TOMCAT }\end{array}$ & 30 & $7.14 \mathrm{E}+11$ & \multicolumn{2}{|c|}{ https://www.psi.ch/en/sls/tomcat/beamline-optics (with multilayer monochromator) } \\
\hline $\begin{array}{l}\text { Canad. LS } \\
\text { BMIT }\end{array}$ & 30 & $5.00 \mathrm{E}+10$ & \multicolumn{2}{|c|}{$\begin{array}{l}\text { https://www.lightsource.ca/beamlines/details/biomedical_imaging_and_therapy_bmitid. } \\
\text { html (assume flux is at } 30 \mathrm{keV} \text { ) }\end{array}$} \\
\hline \multirow{2}{*}{ ESRF ID19 } & 50 & $1.00 \mathrm{E}+12$ & \multirow{2}{*}{\multicolumn{2}{|c|}{ Weitkamp et al. ${ }^{11}$}} \\
\hline & 100 & $1.00 \mathrm{E}+11$ & & \\
\hline \multirow{3}{*}{$\begin{array}{l}\text { Austral. } \\
\text { Synch. } \\
\text { IMBL, } \\
\text { Hutch 2B, } \\
\text { mono }\end{array}$} & 50 & $8.16 \mathrm{E}+11$ & \multirow{6}{*}{\multicolumn{2}{|c|}{$\begin{array}{l}\text { Stevenson et al. }{ }^{12} \\
0.1 \% \text { bandwidth; flux will be much higher for pink beam }\end{array}$}} \\
\hline & 100 & $4.08 \mathrm{E}+11$ & & \\
\hline & 150 & $8.16 \mathrm{E}+10$ & & \\
\hline \multirow{3}{*}{$\begin{array}{l}\text { Austral. } \\
\text { Synch. } \\
\text { IMBL, } \\
\text { Hutch 3B, } \\
\text { mono }\end{array}$} & 50 & $5.49 \mathrm{E}+10$ & & \\
\hline & 100 & $2.74 \mathrm{E}+10$ & & \\
\hline & 150 & $5.49 \mathrm{E}+09$ & & \\
\hline \multirow{3}{*}{ Diamond I12 } & 50 & $2.00 \mathrm{E}+11$ & \multirow{3}{*}{\multicolumn{2}{|c|}{$\begin{array}{l}\text { Drakopoulos et al. }{ }^{13} \text {, Fig. } 2 \\
0.1 \% \text { bandwidth }\end{array}$}} \\
\hline & 100 & $4.00 \mathrm{E}+10$ & & \\
\hline & 150 & $7.00 \mathrm{E}+09$ & & \\
\hline \multirow{3}{*}{$\begin{array}{l}\text { Next-gen } \\
\text { CLS, } 30-90 \\
\text { keV }\end{array}$} & 30 & $6.00 \mathrm{E}+09$ & \multirow{3}{*}{\multicolumn{2}{|c|}{ At $4 \mathrm{~m}$ from source point }} \\
\hline & 60 & $1.68 \mathrm{E}+10$ & & \\
\hline & 90 & $2.72 \mathrm{E}+10$ & & \\
\hline \multirow{3}{*}{$\begin{array}{l}\text { Next-gen } \\
\text { CLS, } 60-180 \\
\text { keV }\end{array}$} & 60 & $3.76 \mathrm{E}+09$ & \multirow{3}{*}{\multicolumn{2}{|c|}{ At $4 \mathrm{~m}$ from source point }} \\
\hline & 120 & $1.24 \mathrm{E}+10$ & & \\
\hline & 180 & $2.20 \mathrm{E}+10$ & & \\
\hline \multirow{3}{*}{$\begin{array}{l}\text { Transmission } \\
\text { microfocus } \\
\text { source } \\
\text { (estimate) }\end{array}$} & 20 & $1.40 \mathrm{E}+09$ & $\mathrm{~V}_{\max }=60 \mathrm{kV}$ & \multirow{3}{*}{$\begin{array}{l}\text { Flux in full bandwidth, energy is approximate mean energy of } \\
\text { bremsstrahlung spectrum. Source spot size } 2.5 \mu \mathrm{m} \text { FWHM, } 5 \mathrm{~mm} \\
\text { source to sample, } 21 \mathrm{~mm} \text { sample to detector, } 3.375 \mu \mathrm{m} \text { detector } \\
\text { pixel size. }\end{array}$} \\
\hline & 50 & $2.20 \mathrm{E}+09$ & $\mathrm{~V}_{\max }=100 \mathrm{kV}$ & \\
\hline & 90 & $1.40 \mathrm{E}+09$ & $\mathrm{~V}_{\max }=160 \mathrm{kV}$ & \\
\hline $\begin{array}{l}\text { Liquid Metal } \\
\text { Jet source }\end{array}$ & 20 & $2.40 \mathrm{E}+09$ & \multicolumn{2}{|c|}{$\begin{array}{l}\text { Larsson et al. }{ }^{14} \text {; flux in full bandwidth scaled from } 400 \mathrm{~W} \text { to } 250 \mathrm{~W} ; \mathrm{V}_{\max }=60 \mathrm{kV} \text {; } \\
\text { energy is approximate mean energy of bremsstrahlung spectrum. Source spot size } 8 \mu \mathrm{m} \\
\text { FWHM, } 26 \mathrm{~mm} \text { source to sample, } 5 \mathrm{~mm} \text { sample to detector, } 0.65 \mu \mathrm{m} \text { detector pixel size }\end{array}$} \\
\hline
\end{tabular}


Table 3. Select synchrotron beamlines and laboratory source estimates used for focused flux comparison in Sec. 3.4.

\begin{tabular}{|c|c|c|c|c|}
\hline Beamline / X-ray source & $\begin{array}{l}\text { Energy } \\
{[\mathrm{keV}]}\end{array}$ & $\begin{array}{l}\text { Focal spot } \\
\text { size hor } x \\
\text { vert }[\mu \mathrm{m}]\end{array}$ & $\begin{array}{l}\text { Focused flux } \\
{[\mathrm{ph} / \mathrm{s} /} \\
\left.(\mathbf{1 0 0} \boldsymbol{\mu m})^{2}\right]\end{array}$ & Reference / notes \\
\hline SSRL $10-2 b$ & 22 & $300 \times 600$ & $1.67 \mathrm{E}+11$ & $\begin{array}{l}\text { https://www-ssrl.slac.stanford.edu/content/beam- } \\
\text { lines/bl10-2b } \\
\text { Assume flux is at high end of energy range }\end{array}$ \\
\hline SSRL 11-3 & 12.7 & $3000 \times 150$ & $4.44 \mathrm{E}+10$ & $\begin{array}{l}\text { https://www-ssrl.slac.stanford.edu/ } \\
\text { content/beam-lines/bl11-3 }\end{array}$ \\
\hline ALS 12.2.2 DCM & \multirow{2}{*}{25} & \multirow{2}{*}{$100 \times 100$} & $1.00 \mathrm{E}+11$ & \multirow{2}{*}{ https://als.lbl.gov/beamlines/12-2-2/ } \\
\hline ALS 12.2.2 Multilayer & & & $1.00 \mathrm{E}+13$ & \\
\hline APS 6-ID-B,C & 8 & $1500 \times 150$ & $3.16 \mathrm{E}+11$ & $\begin{array}{l}\text { https://www.aps.anl.gov/Beamlines/ } \\
\text { Directory/Details?beamline_id=10 }\end{array}$ \\
\hline \multirow{2}{*}{ APS 11-ID-B } & 58.66 & $500 \times 500$ & $9.20 \mathrm{E}+10$ & \multirow{2}{*}{$\begin{array}{l}\text { https://www.aps.anl.gov/Beamlines/ } \\
\text { Directory/Details?beamline_id=16 }\end{array}$} \\
\hline & 86.7 & $500 \times 500$ & $2.00 \mathrm{E}+10$ & \\
\hline APS 11-ID-C & 105.6 & $200 \times 200$ & $2.50 \mathrm{E}+10$ & $\begin{array}{l}\text { https://www.aps.anl.gov/Beamlines/ } \\
\text { Directory/Details?beamline id=15 }\end{array}$ \\
\hline APS 13-BM-C & 15 & $350 \times 30$ & $9.52 \mathrm{E}+11$ & $\begin{array}{l}\text { https://www.aps.anl.gov/Beamlines/ } \\
\text { Directory/Details?beamline id=96 }\end{array}$ \\
\hline APS 17-BM-B & 27 & $300 \times 300$ & $6.67 \mathrm{E}+09$ & $\begin{array}{l}\text { https://www.aps.anl.gov/Beamlines/ } \\
\text { Directory/Details?beamline id }=88\end{array}$ \\
\hline APS 18-ID-D & 12 & $150 \times 150$ & $2.67 \mathrm{E}+13$ & $\begin{array}{l}\text { https://www.aps.anl.gov/Beamlines/ } \\
\text { Directory/Details?beamline_id=25 }\end{array}$ \\
\hline APS 33-BM-C & 20 & $900 \times 500$ & $2.22 \mathrm{E}+10$ & $\begin{array}{l}\text { https://www.aps.anl.gov/Beamlines/ } \\
\text { Directory/Details?beamline id }=83\end{array}$ \\
\hline PETRA III P02.1 & 60 & $500 \times 1100$ & $7.27 \mathrm{E}+08$ & $\begin{array}{l}\text { http://photon-science.desy.de/facilities/petra_iii/ } \\
\text { beamlines/p021_powder_diffraction_and_total_ } \\
\text { scattering_beamline/unified_data_sheet/index_eng.html }\end{array}$ \\
\hline $\begin{array}{l}\text { NSLS-II 4-ID 6-circle } \\
\text { endstation }\end{array}$ & 10 & $400 \times 40$ & $3.13 \mathrm{E}+12$ & $\begin{array}{l}\text { https://www.bnl.gov/ps/beamlines/beamline.php?r=4- } \\
\text { ID (selected } 10 \mathrm{keV} \text { for flux data) }\end{array}$ \\
\hline \multirow{3}{*}{ Next-gen CLS, 30-90 keV } & 90 & $112 \times 112$ & $5.10 \mathrm{E}+10$ & \multirow{3}{*}{$\begin{array}{l}\text { Using multilayer-coated KB mirror focusing optics, } 1: 1 \\
\text { focusing geometry }\end{array}$} \\
\hline & 60 & $137 \times 137$ & $4.26 \mathrm{E}+10$ & \\
\hline & 30 & $177 \times 177$ & $2.17 \mathrm{E}+10$ & \\
\hline MuCLS & 15 & $150 \times 150$ & $2.22 \mathrm{E}+09$ & $\begin{array}{l}\text { Estimate based on earlier measurements with } \\
\text { multilayer-coated KB mirror optics at Lyncean. Note } \\
\text { MuCLS does not have KB mirror optics available. }\end{array}$ \\
\hline Incoatec IuS 3.0, Cu & 8 & $100 \times 100$ & $3.90 \mathrm{E}+08$ & \multirow{3}{*}{ https://www.incoatec.de/ims3 } \\
\hline Incoatec IuS 3.0, Mo & 17.5 & $110 \times 110$ & $4.13 E+07$ & \\
\hline Incoatec IuS 3.0, Ag & 22.2 & $100 \times 100$ & $1.50 \mathrm{E}+07$ & \\
\hline $\begin{array}{l}\text { Excillum MetalJet with } \\
\text { Optics, Ga }\end{array}$ & 9.2 & $80 \times 80$ & $7.81 \mathrm{E}+09$ & \multirow{2}{*}{$\begin{array}{l}\text { https://www.excillum.com/products/ } \\
\text { metaljet-sources/metaljet-with-optics/ }\end{array}$} \\
\hline $\begin{array}{l}\text { Excillum MetalJet with } \\
\text { Optics, In }\end{array}$ & 24 & $70 \times 70$ & $6.12 \mathrm{E}+07$ & \\
\hline $\begin{array}{l}\text { Rigaku XtaLAB MM007- } \\
\mathrm{HF} \mathrm{Cu}\end{array}$ & 8 & $100 \times 100$ & $1.68 \mathrm{E}+09$ & https://www.rigaku-oxford.com/mm007.php \\
\hline $\begin{array}{l}\text { Rigaku XtaLAB MM007- } \\
\text { HF Mo (Estimate) }\end{array}$ & 17.5 & $100 \times 100$ & $1.68 \mathrm{E}+08$ & See above, assume Mo source is $10 \mathrm{x}$ less bright than $\mathrm{Cu}$ \\
\hline $\begin{array}{l}\mu \text { focus sealed tube, } \mathrm{Cu} \text { w/ } \\
\text { confocal optic, } 500 \mu \mathrm{m}\end{array}$ & 8 & $500 \times 500$ & $1.00 \mathrm{E}+06$ & Verbal communication \\
\hline $\begin{array}{l}\text { Rotating Anode, } \mathrm{Cu} \mathrm{w} / \\
\text { confocal optic, } 500 \mu \mathrm{m}\end{array}$ & 8 & $500 \times 500$ & $6.00 \mathrm{E}+06$ & Verbal communication \\
\hline $\begin{array}{l}\text { Rotating Anode, Mo w/ } \\
\text { confocal optic, } 500 \mu \mathrm{m}\end{array}$ & 17.5 & $500 \times 500$ & $6.00 \mathrm{E}+05$ & Verbal communication \\
\hline
\end{tabular}




\section{REFERENCES}

[1] Hajima, R., "Status and Perspectives of Compton Sources," Physics Procedia 84, 35-39 (2016).

[2] Jacquet, M., "Potential of compact Compton sources in the medical field," Physica Medica 32(12), 1790-1794 (2016).

[3] Eggl, E., Dierolf, M., Achterhold, K., Jud, C., Günther, B., Braig, E., Gleich, B. and Pfeiffer, F., "The Munich Compact Light Source: initial performance measures," Journal of Synchrotron Radiation 23(5), 1137-1142 (2016).

[4] "Munich Compact Light Source (MuCLS).”, MSB: Munich Compact Light Source, $<$ https://www.bioengineering.tum.de/en/central-building/munich-compact-light-source/> (31 March 2021 ).

[5] Hornberger, B., Kasahara, J., Gifford, M., Ruth, R. and Loewen, R., "A compact light source providing high-flux, quasi-monochromatic, tunable X-rays in the laboratory," Advances in Laboratory-based X-Ray Sources, Optics, and Applications VII, A. Murokh and D. Spiga, Eds., 2, SPIE, San Diego, United States (2019).

[6] Günther, B., Gradl, R., Jud, C., Eggl, E., Huang, J., Kulpe, S., Achterhold, K., Gleich, B., Dierolf, M. and Pfeiffer, F., "The versatile X-ray beamline of the Munich Compact Light Source: design, instrumentation and applications," Journal of Synchrotron Radiation 27(5) (2020).

[7] Thorpe, M. J., Balslev-Clausen, D., Kirchner, M. S. and Ye, J., "Cavity-enhanced optical frequency comb spectroscopy: application to human breath analysis," Opt. Express 16(4), 2387 (2008).

[8] Long, D. A., Fleisher, A. J., Liu, Q. and Hodges, J. T., "Ultra-sensitive cavity ring-down spectroscopy in the midinfrared spectral region," Opt. Lett. 41(7), 1612 (2016).

[9] Mirov, S. B., Fedorov, V. V., Martyshkin, D., Moskalev, I. S., Mirov, M. and Vasilyev, S., "Progress in Mid-IR Lasers Based on Cr and Fe-Doped II-VI Chalcogenides," IEEE J. Select. Topics Quantum Electron. 21(1), 292310 (2015).

[10] Ma, J., Qin, Z., Xie, G., Qian, L. and Tang, D., "Review of mid-infrared mode-locked laser sources in the $2.0 \mu \mathrm{m}-$ $3.5 \mu \mathrm{m}$ spectral region," Applied Physics Reviews 6(2), 021317 (2019).

[11] Weitkamp, T., Tafforeau, P., Boller, E., Cloetens, P., Valade, J.-P., Bernard, P., Peyrin, F., Ludwig, W., Helfen, L., Baruchel, J., Denecke, M. and Walker, C. T., "Status and evolution of the ESRF beamline ID19," presented at X-RAY OPTICS AND MICROANALYSIS: Proceedings of the 20th International Congress, 2010, Karlsruhe (Germany), 33-38.

[12] Stevenson, A. W., Crosbie, J. C., Hall, C. J., Häusermann, D., Livingstone, J. and Lye, J. E., "Quantitative characterization of the X-ray beam at the Australian Synchrotron Imaging and Medical Beamline (IMBL),"J Synchrotron Rad 24(1), 110-141 (2017).

[13] Drakopoulos, M., Connolley, T., Reinhard, C., Atwood, R., Magdysyuk, O., Vo, N., Hart, M., Connor, L., Humphreys, B., Howell, G., Davies, S., Hill, T., Wilkin, G., Pedersen, U., Foster, A., De Maio, N., Basham, M., Yuan, F. and Wanelik, K., "I12: the Joint Engineering, Environment and Processing (JEEP) beamline at Diamond Light Source," Journal of Synchrotron Radiation 22(3) (2015).

[14] Larsson, D. H., Vågberg, W., Yaroshenko, A., Yildirim, A. Ö. and Hertz, H. M., "High-resolution short-exposure small-animal laboratory x-ray phase-contrast tomography," Scientific Reports 6(1) (2016).

[15] D. Bleiner, The Science and Technology of X-ray Lasers: A 2020 Update Proc. SPIE 11886, 1188602 (2021) 